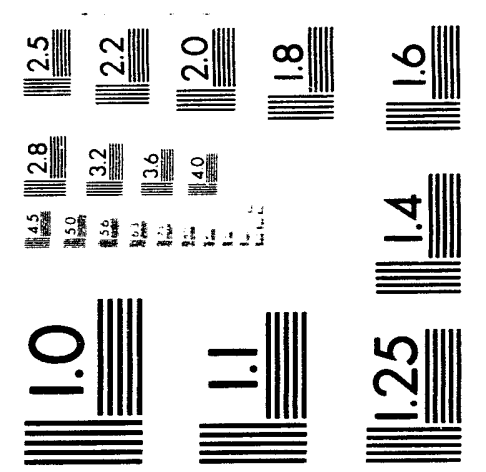



Distribution Category:

Mathematics and

Computer Science (UC-405)

\section{ARGONNE NATIONAL LABORATORY \\ 9700 South Cass Avenue \\ Argonne, IL 60439}

ANL-93/34

\section{Periodized Wavelets}

by

George Schlossnagle, ${ }^{\dagger} *$ Juan Mario Restrepo, and Gary K. Leaf

Mathematics and Computer Science Division

December 1993

Support for this work was provided by the Atmospheric and Climate Research Division and the Office of Scientific Computing, U.S. Department of Energy, under Contract W-31-109-Eng-38.

†Participant in the Summer 1993 Student Research Participation Program. This program is coordinated by the Division of Educational Programs.

${ }^{*}$ Johns Hopkins University, Baltimore, MD, U.S.A. 


\section{Contents}

Abstract 1

[1]. Introduction 1

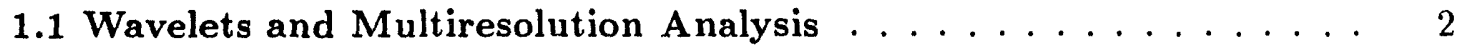

1.2 Other Properties of Wavelets . . . . . . . . . . . . . 3

[2]. Periodized Wavelets 4

[3]. Approximation Results and Methods 6

[4]. Methods for Computing Connection Coefficients 13

4.1 Formation of Homogeneous Relations . . . . . . . . . . . . . . 14

4.2 Generating Inhomogeneous Relations . . . . . . . . . . . 15

Appendix: Tables of Connection Coefficients $\quad 17$

$\begin{array}{ll}\text { References } & 19\end{array}$ 


\title{
PERIODIZED WAVELETS
}

\author{
George Schlossnagle, Juan Mario Restrepo, Gary K. Leaf
}

\begin{abstract}
The properties of periodized Daubechies wavelets on $[0,1]$ are detailed and contrasted against their counterparts which form a basis for $L^{2}(\mathbb{R})$. Numerical examples illustrate the analytical estimates for convergence and demonstrate by comparison with Fourier spectral methods the superiority of wavelet projection methods for approximations. The analytical solution to inner products of periodized wavelets and their derivatives, which are known as connection coefficients, is presented, and several tabulated values are included.
\end{abstract}

\section{[1]. Introduction.}

Wavelets are finding a well-deserved niche in such areas of applied mathematics and engineering as approximation theory, signal analysis, and projection techniques for the solution of differential equations. While the concept of wavelets is not conceptually new [1] [2] [3], the past fifteen years have produced much of the theoretical underpinnings for the concept, as well as the generation of new wavelet families and the exploration of their potential in various areas of applied science [1] [2] [3] [4] [5].

Wavelets have several advantages: (1) they have compact support or exponentially decaying support; (2) their continuity properties may easily be increased, albeit at the expense of a larger domain of support; (3) for a given spline order, a complete basis may easily be generated by simple recurrence relations; (4) in the context of projection techniques, their convergence properties are as good as or better than Fourier methods, and they permit the analysis of extremely local functional behavior without the need for windowing and with little or no bias from global behavior; and (5) the manner in which the space is broken down into a family of multiply-enclosed subspaces enables spatial or temporal function multiresolution analysis.

To date, research has focused on compactly supported wavelets [2]; basic wavelets [3]; and smooth, exponentially decaying wavelets [6]. All of these wavelet families form a basis for $L^{2}(\mathbb{R})$. Work is currently under way on the generation of wavelets that are capable of spanning other classes of function space and/or domain. For example, Meyer [3] has shown how compactly supported wavelets can be made to form a basis for $L^{2}([0,1])$. He generates the periodized family by wrapping the $L^{2}(\mathbb{R})$ basis on a torus.

This report shows in detail how such a wrapping procedure is accomplished using Daubechies wavelets. The properties of the resulting wavelets on $L^{2}([0,1])$ are then contrasted with more standard Daubechies wavelets, which are now extremely popular in the signal processing community. This study also compares the convergence characteristics of periodized wavelet interpolation of functions with the characteristics of the Fourier spectral method. 
The final section of this report is devoted to a derivation and numerical calculation of connection coefficients involving periodized Daubechies wavelets. Connection coefficients are matrix structures that result from the evaluation of inner products of the form

$$
\int \varphi_{j, k_{0}}^{\left(d_{0}\right)} \varphi_{j, k_{1}}^{\left(d_{1}\right)} \ldots \varphi_{j, k_{n}}^{\left(d_{n}\right)} d x
$$

where $d_{i}$ is the number of differentiation with respect to $x$ of the scaling function $\varphi=$ $\varphi(x)$. Inner products arise naturally in the context of the Galerkin solution of differential equations. The name for these inner products was coined by Latto et al. [7], who developed a computational method that avoids the pitfalls of quadrature techniques. We use the technique of Latto et al. to calculate connection coefficients for the periodized Daubechies wavelets. A sample table of these cocfficients appears in the appendix. Also included in the appendix is information on how to obtain these and other tabulated values of connection coefficients from the Mathematics and Computer Science Division at Argonne National Laboratory.

\subsection{Wavelets and Multiresolution Analysis.}

The value of wavelets hinges on their ability to perform multiresolution analysis. A multiresclution analysis is a nested sequence

$$
V_{0} \subset V_{1} \subset \ldots \subset L^{2}(\mathbb{R})
$$

satisfying the following properties:

(1) $\bigcap_{j \in \mathbb{Z}} V_{j}=0$.

(2) $\operatorname{clos}_{L^{2}}\left(\bigcup_{j \in \mathbb{Z}} V_{j}\right)=L^{2}(\mathbb{R})$.

(3) $f(x) \in V_{j} \Leftrightarrow f(2 x) \in V_{j+1}$.

(4) There is a function $\varphi \in V_{0}$ such that $\left\{\varphi_{0, k}(x)=\varphi(x-k)\right\}_{k \in \mathbb{Z}}$ forms a Riesz basis for $V_{0}$.

The term $\varphi$ is called the mother scaling function since, from (3), there exists $\left\{h_{k}\right\} \in l^{2}$ such that

$$
\varphi(x)=\sqrt{2} \sum_{k \in \mathbb{Z}} h_{k} \varphi(2 x-k) .
$$

This relation, called the scaling relation, will also hold for $\varphi(2 x)$ and, by induction, for $\varphi\left(2^{j} x\right)$. In accordance with the notation in (4), we denote the translates and dilations of $\varphi$ by

$$
\varphi_{j, k}(x)=2^{\frac{j}{2}} \varphi\left(2^{j} x-k\right) .
$$

The set $\left\{\varphi_{j, k}\right\}_{k \in Z}$ forms a Riesz basis for $V_{j}$. We define $W_{j}$ to be the orthogonal complement of $V_{j}$ with respect to $V_{j+1}$. Just as $V_{j}$ is spanned by dilations and translations of the mother scaling function, so are the $W_{j}$ 's spanned by translations and dilations of the mother wavelet. The mother wavelet is defined by

$$
\psi(x)=\sqrt{2} \sum_{k}(-1)^{k-1} h_{-k+1+2 M} \varphi_{1, k}(x)
$$


with $M$ a particular integer. Daubechies [2] constructed compactly supported wavelets and scaling functions using a finite set of nonzero $\left\{h_{k}\right\}_{k=0}^{N-1}$ scaling parameters with $N=2 M$

and $\sum_{k=0}^{N-1} h_{k}=\sqrt{2}$. With these scaling parameters, the recursion formulas generate the desired orthogonal wavelets and scaling functions with $\operatorname{supp}(\varphi)=[0, N-1]$. Henceforth, these will be the wavelets we shall use, which we refer to as $D_{N}$ or Daubechies wavelets of order $N$. Values for $\varphi$ are calculated using the scaling relation as indicated in the following procedure. First, the values it takes are determined at integer points. Then at the dyadic rationals at level 1 (the dyadic rationals at level $\mathrm{j}$ are $\mathbb{D}_{j}=\left\{\frac{k}{2^{j}}\right\}_{k \in \mathbb{Z}}$ ). Using that information, we calculate the values at $\mathbb{D}_{2}$ and so on, until $\varphi$ is defined at the dyadic rationals at all levels. Since the dyadics are dense in the reals, we simply extend $\varphi$ continuously to $\mathbb{R}$. This procedure creates a function that is continuous but not differentiable for $N=4$, differentiable but not twice differentiable for $N=6$, and with increasing regularity for increasing $N$ [2]. The nature of the scaling relation guarantees that $\varphi$ will be discontinuous in some derivative [8]. This procedure is easily accomplished computationally.

Another pleasing feature of these wavelets is their compact support. Whereas Fourier methods return global results, with compactly supported wavelets one can easily analyze short-lived events or pulses. Wavelet projection methods avoid distortion that might result from a local analysis with a windowed Fourier transform. As we shall see in the next section, compact support also makes the periodization of these wavelets an elegant process.

\subsection{Other Properties of Wavelets.}

In addition to items (1)-(4) mentioned above, wavelets have a number of other interesting properties. These will be given without proof. For further details, see Daubechies [2] or Chui $[1]$.

(5) $\left\{\varphi_{j, k}\right\}_{j \geq 0, k \in \mathbb{Z}}$ is an orthonormal basis for $L^{2}(\mathbb{R})$.

(6) $V_{j+1}=V_{j} \oplus W_{j}$

(7) $L^{2}(\mathbb{R})=\operatorname{clos}_{L^{2}}\left(V_{0} \bigoplus_{j=0}^{\infty} W_{j}\right)$.

(8) $\left\{\varphi_{0, k}, \psi_{j, k}\right\}_{j \geq 0, k \in \mathbb{Z}}$ is an orthonormal basis for $L^{2}(\mathbb{R})$.

(9) $\left\{\varphi_{j, k}, \psi_{l, k} ; 0 \leq j \leq J \leq l, k \in \mathbb{Z}\right\}$ is an orthonormal basis for $L^{2}(\mathbb{R})$.

(10) $\int_{-\infty}^{\infty} \varphi(x) d x=1$.

(11) $\sum_{k \in \mathbb{Z}} \varphi_{0, K}=1$.

(12) $\int_{-\infty}^{\infty} \psi(x) x^{k} d x:=0: k=0, \ldots, M-1$.

(13) $\left\{x^{k}\right\}_{k=0}^{N-1} \in V_{0}$.

Item (6) is really the heart of multiresolution analysis and provides wavelet-based analysis with a distinctly different resolving quality in contrast to spectral methods: to go to a higher resolution of spatial scale, one simply adds on the next wavelet level (the next $W_{j}$ ) as implied by item (6). At some given level (say, with a representation in $V_{j}$ ), the multiresolution property guarantees all spatial scale information at all coarser levels. In contrast, with Fourier methods, information about one frequency gives no information about other frequencies. 


\section{[2]. Periodized Wavelets.}

The wavelets developed above are defined on $\mathbb{R}$. For inany applications, however, wavelets defined on a periodic domain are needed. In erestingly, the wavelets defined above can be periodized with a Poisson summation technique to give periodic wavelets [2] that possess many of the same properties of their nonperiodic kin. Moreover, for large enough $j$, the periodization of $\varphi_{j, k}$ and $\psi_{j, k}$ is identical to their nonperiodic forms except for wrapping around the edges of the domain; and for large enough $j$, this too can be reduced to the nonperiodic case for most calculations. As would be expected, many of the above-mentioned properties are preserved in the periodic case as a result of the construction by the "scaling" property of the nonperiodic functions and their compact support.

The wavelets are periodized as follows:

$$
\hat{\varphi}_{j, k}(x) \equiv \sum_{l \in \mathbb{Z}} \varphi_{j, k}(x-l) \text { and } \hat{\psi}_{j, k}(x)=\sum_{l \in \mathbb{Z}} \varphi_{j, k}(x-l) .
$$

By construction $\hat{\varphi}$ and $\hat{\psi}$ are periodic and are well defined on $[0,1]$ since $\varphi$ and $\psi$ have compact support. Note that $\varphi_{j, k}=\varphi_{j, k^{\prime}}$ if $k \equiv k^{\prime} \bmod \left(2^{j}\right)$. Thus we shall restrict our attention to $0 \leq k<2^{j}$. The same holds for the $\psi$ 's. In what follows, the properties of the periodic wavelets will be investigated in detail.

Periodized wavelet bases are not generated in quite the same way as the nonperiodic versions. In the nonperiodic case, bases are generated by repeated translation and dilation of the mother functions; but this approach is not possible in the periodic case, since periodization does not commute with dilation. Therefore, the wavelet must be first dilated, then periodized. Although proof can be shown for the general case, we shall instead show that the elements in $V_{j}$ for $j \leq 0$ are all constant functions. If dilation commuted with periodization, this would not be true.

Proposition. For $j \leq 0, \varphi_{j, k}=2^{\frac{-j}{2}}$.

Proof. Since

$$
\varphi_{j, k}=2^{\frac{i}{2}} \varphi\left(2^{j} x-k\right)
$$

then

$$
\begin{aligned}
\hat{\varphi}_{j, k} & =\sum_{l \in \mathbb{Z}} 2^{\frac{i}{2}} \varphi\left(2^{j}(x-l)-k\right) \\
& =2^{\frac{j}{2}} \sum_{b=0}^{2^{-j}} \sum_{l \in \mathbb{Z}} \varphi\left(2^{j} x-\left(l+\frac{b}{2^{-j}}\right)-k\right) .
\end{aligned}
$$

Letting $y=2^{j} x$ and summing over $l$, we obtain

$$
\hat{\varphi}_{j, k}=2^{\frac{j}{2}} \sum_{b=0}^{2^{-j}-1} 1=2^{\frac{-j}{2}} .
$$

$\diamond$

The most important property to be carried over to the periodized case is, of course, that the new functions form an orthonormal basis for $L^{2}[0,1]$. 
Proposition. $\left\{\varphi_{j, k}: j \geq 0,0 \leq k<2^{j}\right\}$ forms an orthonormal basis for $L^{2}[0,1]$.

Definition: $\langle f, g\rangle=\int f(x) g(x) d x$, the standard $L^{2}$ inner product.

Proof. We begin by showing that $\left\langle\hat{\varphi}_{j, k}, \hat{\varphi} j^{\prime}, k^{\prime}\right\rangle=0$ :

$$
\left\langle\hat{\varphi}_{j, k}, \hat{\varphi} j^{\prime}, k^{\prime}\right\rangle=2^{\frac{i+j^{\prime}}{2}} \int_{0}^{1} \sum_{l, l^{\prime} \in \mathbb{Z}} \varphi\left(2^{j}(x-l)-k\right) \varphi\left(2^{j^{\prime}}\left(x-l^{\prime}\right)-k^{\prime}\right) d x .
$$

Let $y=x-l$, so that

$$
\begin{aligned}
\left\langle\hat{\varphi}_{j, k}, \hat{\varphi} j^{\prime}, k^{\prime}\right\rangle & =2^{\frac{i+j^{\prime}}{2}} \int_{0}^{1} \sum_{l, l^{\prime} \in \mathbb{Z}} \varphi\left(2^{j}(x-l)-k\right) \varphi\left(2^{j^{\prime}}\left(x-l^{\prime}\right)-k^{\prime}\right) d x \\
& =2^{\frac{i+j^{\prime}}{2}} \int_{-l^{\prime}}^{1-l^{\prime}} \sum_{l, l^{\prime} \in \mathbb{Z}} \varphi\left(2^{j} y+2^{j}\left(l-l^{\prime}\right)-k\right) \varphi\left(2^{j^{\prime}} y-k^{\prime}\right) d x \\
& =2^{\frac{i+j^{\prime}}{2}} \sum_{r \in \mathbb{Z}} \int_{-\infty}^{\infty} \varphi\left(2^{j} y+2^{j} r-k\right) \varphi\left(2^{j^{\prime}} y-k^{\prime}\right) d x \\
& =\sum_{r \in \mathbb{Z}}\left\langle\varphi_{j, k+2^{j} r}, \varphi_{j^{\prime}, k^{\prime}}\right\rangle=\delta_{j j^{\prime}} \delta_{k k^{\prime}},
\end{aligned}
$$

with $l-l^{\prime}=r$. Thus $\hat{\varphi}_{j, k}$ and $\hat{\varphi}_{j^{\prime}, k^{\prime}}$ are orthonormal. Next we show that they form a basis for $L^{2}[0,1]$.

Choose an arbitrary $f \in L^{2}[0,1]$. Now consider

$$
\tilde{f}(x)=f(x) x \in[0,1]=0 x \notin[0,1] .
$$

$\tilde{f} \in L^{2}(\mathbb{R})$ and $\left\{\varphi_{j, k}\right\}$ form an orthonormal basis for $L^{2}(\mathbb{R})$, so we have $\tilde{f}=\sum_{\substack{0 \leq j \\ k \in \mathbb{Z}}}\left\langle\tilde{f}, \varphi_{j, k}\right\rangle$ which, when periodized, becomes

$$
f(x)=\sum_{l \in \mathbb{Z}} \tilde{f}(x-l)=\sum_{i \in \mathbb{Z}} \sum_{\substack{0 \leq j \\ k \in \mathbb{Z}}}\left\langle\tilde{f}, \varphi_{j, k}\right\rangle=\sum_{\substack{0 \leq j \\ k \in \mathbb{Z}}}\left\langle\tilde{f}, \hat{\varphi}_{j, k}\right\rangle .
$$

This final result is actually a finite sum since, for $k \geq 2^{j}$ and $k \leq 1-N, \operatorname{supp}\left(\varphi_{j, k}\right) \cap$ $\operatorname{supp}(\tilde{f})=\emptyset$. Thus $f$ has a representation in the periodized wavelets.

$\diamond$

The proof that $\left\{\hat{\varphi}_{j, k}, \hat{\psi}_{j^{\prime}, k}: j^{\prime} \geq j \geq 0,0 \leq k<2^{j}\right\}$ also form an orthonormal basis is nearly identical. Since $\varphi(x)=\sum_{k=0}^{N-1} h_{k} \varphi(2 x-k)$, we can periodize both sides to get

$$
\begin{aligned}
& \hat{\varphi}(x)=\sum_{l \in \mathbb{Z}} \varphi(x-l)=\sum_{l} \sum_{k} h_{k} \varphi(2(x-l)-k)=\sum_{k} \sum_{l} h_{k} \varphi(2(x-l)-k) \\
& =\sum_{k} h_{k} \hat{\varphi}_{1, k}(x) .
\end{aligned}
$$


Thus we have an orthonormal basis that still has a scaling relation. This means that in comparison with the nonperiodic case, we have a chain of spaces $\hat{V}_{0} \subset \hat{V}_{1} \subset \ldots \subset L^{2}[0,1]$ with the following properties:

$$
\bigcup_{j \geq 0} \hat{V}_{j}=L^{2}[0,1] \text { with } V_{j}=\operatorname{span}\left\{\hat{\varphi}_{j, k}\right\}_{k=0}^{2^{j}-1}
$$

(15) $f(x) \in V_{j} \Leftrightarrow f(2 x) \in V_{j+1}$.

(16) By defining $\hat{W}_{j}=\operatorname{span}\left\{\hat{\psi}_{j, k}\right\}_{k=0}^{2^{j}-1}$ we see that $\hat{W}_{j}$ is the orthogonal complement of $\hat{V}_{j}$ in $\hat{V}_{j+1}$. So then $\operatorname{clos}\left(V_{0} \bigoplus_{j=0}^{\infty} W_{j}\right)=L^{2}[0,1]$

Clearly there are some differences between the properties of the periodic case and the nonperiodic case. While they are both multiresolution spaces, the basis functions in the nonperiodic case are all formed by translations and dilations of the mother scaling function $\varphi$, while in the periodic case it is often impossible to derive $\hat{\varphi}_{j+1}$ from $\hat{\varphi}_{j}$ (for example, consider $\hat{\varphi}_{1}$ and $\hat{\varphi}_{0}$, the latter is a constant function and thus unable to represent the former). It turns out, however, that there is no relation between $\hat{\varphi}_{j+1}$ and $\hat{\varphi}_{j}$ only for very small $j$. For $j$ suitably large, the periodic case actually looks exactly the same as the nonperiodic case. This result is formalized as follows.

Proposition. For $j \geq \log _{2}(N-1), \hat{\varphi}_{j, 0}=\varphi_{j, 0}$, where $\hat{\varphi}$ is extended to $\mathbb{R}$ by setting it to 0 away from the unit interval.

Proof. $\operatorname{supp}\left(\varphi_{j, 0}\right)=\left[0,2^{-j}(N-1)\right]$, so for $j \geq \log _{2}(N-1), \operatorname{supp}\left(\varphi_{j, 0}\right)=[0, \beta], \beta \leq 1$. Thus $\hat{\varphi}_{j, 0}(x)=\sum_{l \in \mathbb{Z}} \varphi_{j, 0}(x-l)=\varphi_{j, 0}$.

$$
\diamond
$$

Thus, for large enough $j$, the periodization will affect the functions only by "wrapping them around" the edges of the domain. This is also a strong argument for using the scaling functions as trial-and-test functions when using periodized wavelets. By choosing $j$ well, calculations can be performed as in the nonperiodic case; if desired, a multiresolution can then be performed easily. Calculations will not be so simple if $\hat{V}_{0} \bigoplus_{k=1}^{j} \hat{W}_{k}$ is used as a test space, since for low $k$ the basis for $\hat{W}_{k}$ is not equivalent to the basis for $W_{k}$.

\section{[3]. Approximation Results and Methods.}

A function $f \in L^{2}[0,1]$ may be projected into the wavelet basis and expressed as $f=$ $\sum_{k} a_{k_{j}} \hat{\varphi}_{j, k}$, where $a_{k_{j}}=\left\langle f, \hat{\varphi}_{j, k}\right\rangle$. Since the calculation of $a_{j_{k}}$ is usually hard to evaluate analytically, numerical methods must be employed. In [9], a method was developed by using Taylor series expansions to approximate $f$. The method requires use of the moment equations to make $\mathcal{O}\left(h^{n}\right)$ approximations for $f \in C^{n}$. Unfortunately, in applications such as signal processing or any area where only a finite number of samples of $f$ are provided, this method fails or requires interpolation. As an alternative, a function $f$ given as samples on $\mathbb{D}_{j} \cap[0,1]$ may be approximated by a function $\tilde{f} \in \hat{V}_{j}$.

Define the samples of $f$ as $\underset{\rightarrow}{f} \in \mathbb{R}$, with the $k$-th component of $\underset{f}{f}=f\left(\frac{k-1}{2^{j}}\right)$. Construct $\hat{\varphi}_{j, k}$ from $\hat{\varphi}_{j, k}$ as shown previously. This yields, for $j>\log _{2}(N-2)$, a linearly independent spanning set for $\mathbb{R}^{2^{j}}$. Further, since $\operatorname{supp}\left(\hat{\varphi}_{j, 0}\right)=\left[0,2^{-j}(N-1), \hat{\varphi}_{j, k}\right.$ takes only $N-2$ valuess 
on $\mathbb{D}_{j}$ and $\hat{\varphi}_{j, k}$ is just the $k$-th forward cyclic permutation of the elements of $\hat{\varphi}_{j, 0}$. The problem is thus reduced to finding the unique representation of $\underset{\rightarrow}{f}$ in terms of $\left\{\hat{\varphi}_{j, k}\right\}_{0 \leq k<2^{j}}$, which is simply the solution of

$$
\stackrel{\vec{v}}{\rightarrow}=\stackrel{f}{\longrightarrow}
$$

where $A$ is the very sparse transformation matrix from the standard basis to the $\underline{\hat{\varphi}}$ basis. Define $\tilde{f}(x)=\sum_{k} \underset{\sim}{\rightarrow}(k) \hat{\varphi}_{j, k}(x)$. By construction, $\left.\tilde{f}\right|_{\mathbb{D}_{j}}=f$.

In summary, this method involves solving the inverse problem with a sparse matrix, and results in a function $\tilde{f}$ with $\left.\tilde{f}\right|_{\mathbb{D}_{j}}=f$. In essence, $\tilde{f}$ will be equal to $f$ at all the sampled values.

We next show the manner in which the periodized wavelets may be used in the context of functional approximation. Since the wavelets form an orthonormal basis, the orthogonal projection operators onto $V_{j}$ and $W_{j}$ are defined respectively as

$$
P_{j}(f)=\sum_{k=0}^{2^{j}-1}\left\langle f, \hat{\varphi}_{j, k}\right\rangle, Q_{j}(f)=\sum_{k=0}^{2^{j}-1}\left\langle f, \hat{\psi}_{j, k}\right\rangle .
$$

As we have already seen, periodized wavelets provide a basis for $L^{2}[0,1]$ so we have $\| f-$ $P_{j} f \|_{2} \rightarrow 0$, as $j \rightarrow \infty$. This is a property of any orthonormal basis of $L^{2}$, but this particular periodized basis has some additional properties.

Theorem. If $f$ is a continuous function on the torus, then $\left\|f-P_{j} f\right\|_{\infty} \rightarrow 0$ as $j \rightarrow \infty$.

Proof. We begin the proof by showing that our projection operator is bounded. $P_{j}$ is an integral operator of the form $P_{j} f(x)=\int_{0}^{1} \sum_{k=0}^{2^{j}-1} \hat{\varphi}_{j, k}(y) \hat{\varphi}_{j, k}(x) f(y) d y$. Thus,

$$
\begin{aligned}
\left\|P_{j}\right\|_{\infty} & \leq \sup _{x \in[0,1]} \int_{0}^{1}\left|\sum_{k=0}^{2^{j}-1} \hat{\varphi}_{j, k}(y) \hat{\varphi}_{j, k}(x)\right| d y \\
& \leq \sup _{x \in[0,1]} \mid \sum_{k=0}^{2^{j}-1} \hat{\varphi}_{j, k}(x)\|\| \hat{\varphi}_{j, k} \|_{\infty}\left[2^{-j}(N-1)\right] \\
& \leq \sup _{x \in[0,1]} 2^{\frac{j}{2}}\left|\sum_{k=0}^{2^{j}-1} \hat{\varphi}\left(2^{j} x-k\right)\right| 2^{\frac{j}{2}}\|\hat{\varphi}\|_{\infty} 2^{\frac{j}{2}}\left[2^{-j}(N-1)\right] \\
& \leq \sup _{x \in[0,1]} \mid \sum_{k=0}^{2^{j}-1} \hat{\varphi}\left(2^{j} x-k\right)\|\hat{\varphi}\|_{\infty}(N-1) .
\end{aligned}
$$

Now, $\left|\sum_{k=0}^{2^{j}-1} \hat{\varphi}\left(2^{j} x-k\right)\right|<(N-1)\|\hat{\varphi}\|_{\infty}$ since for $j \geq \log _{2}(N-1)$ there are at most $(N-1) k$ 's such that for a given $x,\{x\} \cap \operatorname{supp}\left(\hat{\varphi}_{j, k}\right) \neq \emptyset$. Hence, we have

$$
\left\|P_{j}\right\|_{\infty} \leq\|\hat{\varphi}\|_{\infty}^{2}(N-1)^{2} \text {. }
$$


If we take $f \in \bigcup_{j \in \mathbb{N}} V_{j}$, then $\exists J$ such that $\forall j \geq J, Q_{j} f=0$. Thus, $P_{j} f=f$ for $j \geq J$. $\bigcup_{j \in \mathbb{N}} V_{j}$ is dense in $L^{2}[0,1]$ which is dense in $C(\mathbb{T})$, continuous functions of period 1 on the unit interval, Finally, by the boundedness of $P_{j}$, the theorem follows. $\diamond$

Theorem. (Daubechies) If $f \in L^{1}[0,1]$, then $\left\|f-P_{j} f\right\|_{1} \rightarrow 0$ as $j \rightarrow \infty[2]$.

Proof. Since $L^{1}[0,1] \subset(C[0,1])^{\star}$, we have

$$
\begin{aligned}
\left\|P_{j} f\right\|_{1} & =\sup \left\{\left|\left\langle P_{j} f, g\right\rangle\right| ; g \text { continuous, }\|g\|_{\infty} \leq 1\right\} \\
& =\sup \left\{\left|\left\langle f, P_{j} g\right\rangle\right| ; g \text { continuous, }\|g\|_{\infty} \leq 1\right\} \\
& \leq\|f\|_{1}\left\|P_{j} g\right\|_{\infty} .
\end{aligned}
$$

$\left\|P_{j}\right\|_{\infty}$ is bounded by the previous theorem. Since $\bigcup_{j \in \mathbb{N}} V_{j}$ is dense in $L^{2}[0,1]$, which is also dense in $L^{1}[0,1]$, the uniform bound on $P_{j}$ is sufficient to prove our result. $\diamond$

These two results are strong, in contrast to the convergence properties of Fourier functional approximations. In fact it has been shown that the continuous functions whose Fourier series do not uniformly converge are dense in $C(\mathbb{T})[10]$. In this sense, wavelets provide a much more general basis than Fourier bases and hence have potentially broader applications.

These results suggest that wavelets should do a better job at pointwise approximation, especially for continuous functions. In Figures 1-4, we illustrate how wavelet and Fourier functional approximations compare with each other. The figures are graphs of the pulse function $P(x)=e^{-1750\left(x-\frac{1}{2}\right)}$ and the step function $S(x)=\Xi_{\left[0, \frac{1}{2}\right]}$ against a 16 -term $\left(V_{4}\right)$ $D_{6}$ discrete wavelet approximation and against a 16-term discrete Fourier interpolation. These figures clearly show that for the $C^{\infty}$ pulse function, the wavelets provide a much closer pointwise approximation. In fact, the only significant overshoot by the wavelets is at the base of the pulse. In contrast, the Fourier approximation shows a considerable amount of aliasing spread over the entire domain. Overshoot on the step function is confined to a neighborhood of the discontinuity. It is possible to resolve this inaccuracy with the wavelet techniques while maintaining a low number of approximating terms by continuing to take coarse approximations away from the discontinuity and projecting onı a finer scale in a neighborhood of the discontinuity. From these graphs we conclude that the finite-term wavelet functional interpolation is superior to its Fourier counterpart in approximating functions that contain a great deal of local information; moreover, it is better able to capture function discontinuities. 


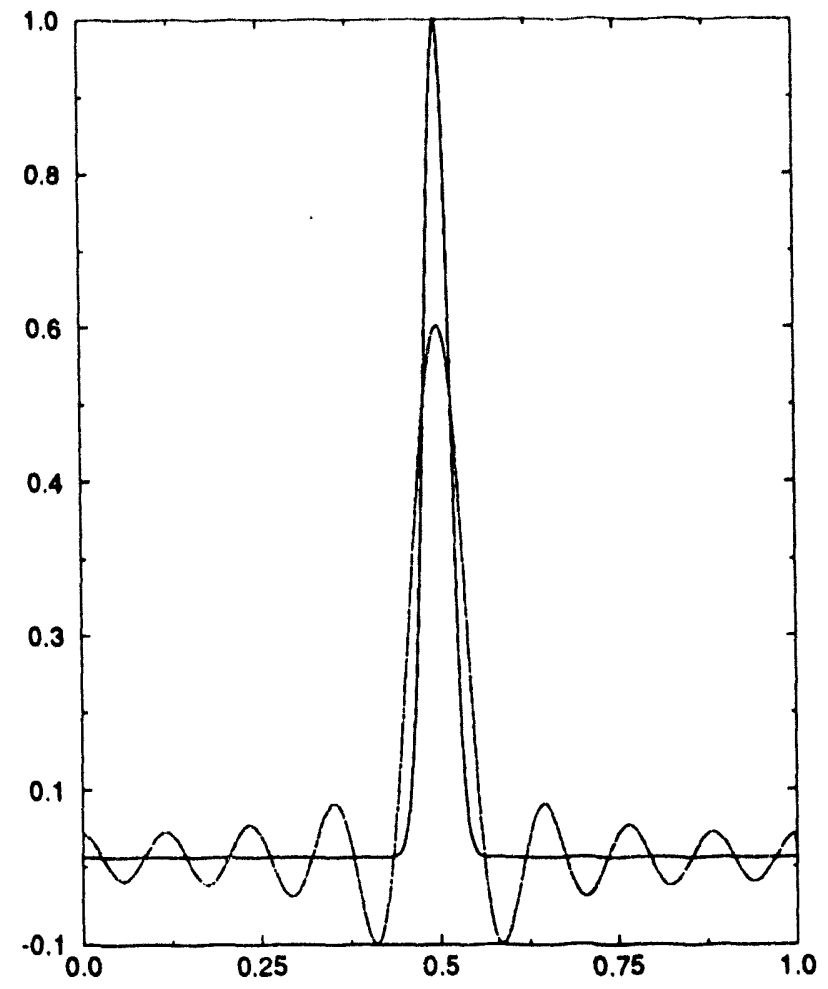

Figure 1. 16-term Fourier approximation to $P(x)$

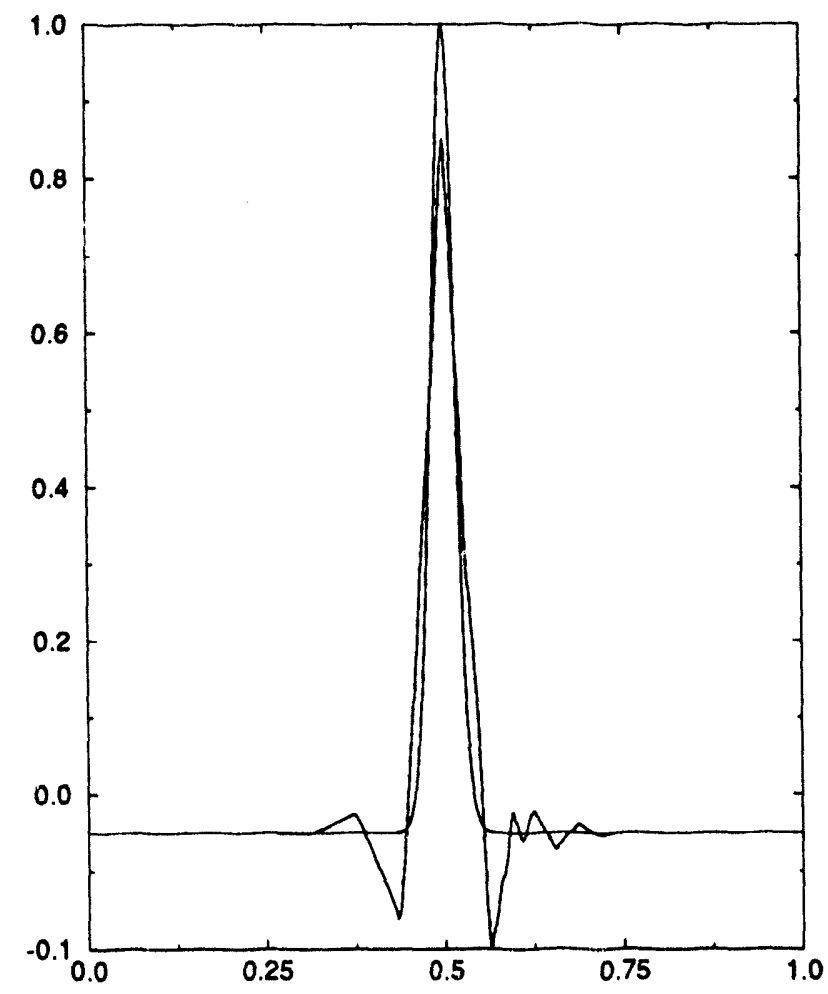

Figure 2. $V_{4}$ wavelet approximation to $P(x)$ 


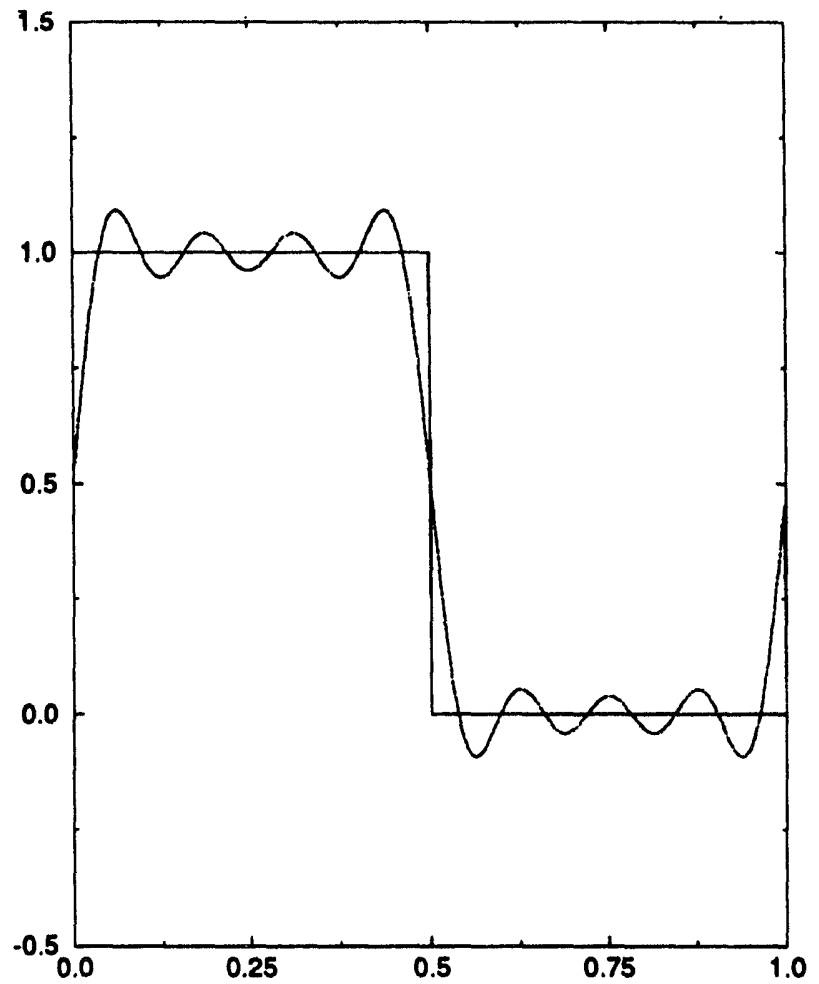

Figure 3. 16-term Fourier approximation to $S(x)$

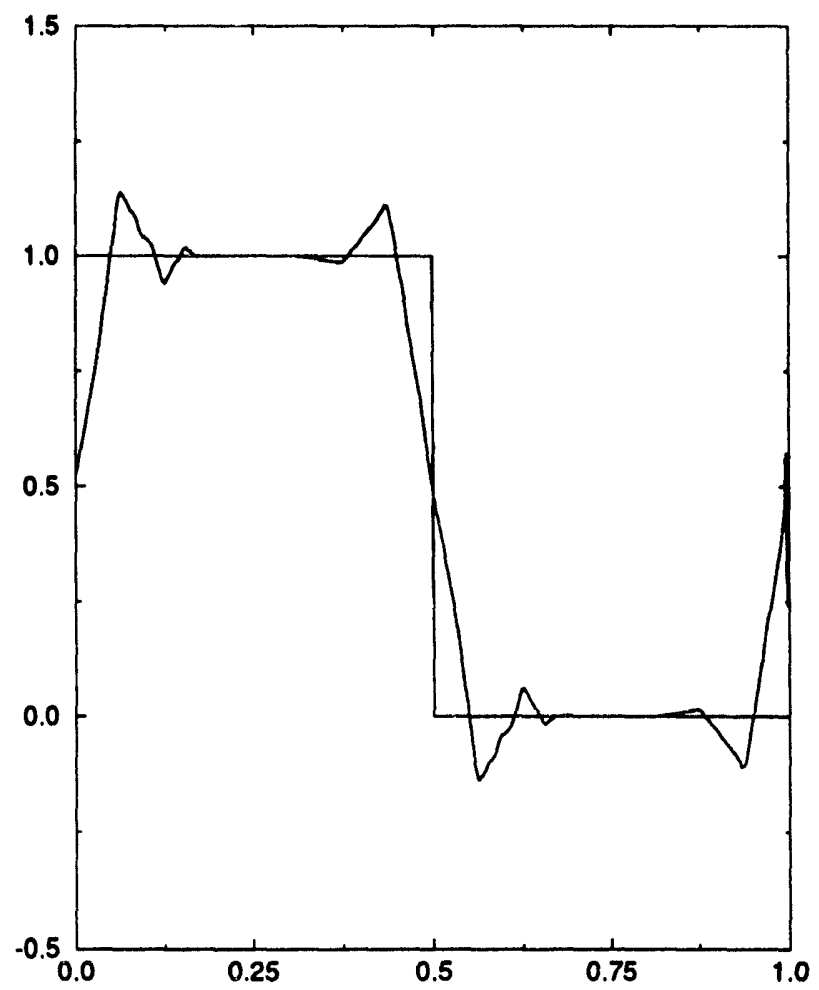

Figure 4. $V_{4}$ wavelet approximation to $S(x)$ 
To measure the error to which a truncated projection will approximate a desired function, we shall estimate its convergence. A natural choice of norms with which to measure convergence is the Sobolev norms. The sth Sobolev norm of a function $f$ is defined as

$$
\|f\|_{H^{\bullet}}=\left(\int\left(1+x^{2}\right)^{s} \hat{f}^{2}(x) d x\right)^{\frac{1}{2}}
$$

where $H^{s}$ consists of those functions whose $s$ th Sobolev norm exists and is finite. In [2] Daubechies states that the norm for $H^{s}$ is equivalent to

$$
\|f\|_{H^{\cdot}[0,1]}=\left(\sum_{\substack{j \geq 0 \\ 0 \leq k<2^{j}}}\left(1+2^{2 j s}\right)\left\langle f, \hat{\psi}_{j, k}\right\rangle^{2}\right)^{\frac{1}{2}}
$$

Using this result, we easily find a bound for $\left\|f-P_{p} f\right\|_{2}$. For $f \in H^{\bullet}[0,1]$,

$$
\begin{aligned}
\left\|f-P_{p} f\right\|_{2} & =\left\|\sum_{j \geq p 0 \leq k<2^{j}}\left\langle f, \hat{\psi}_{j, k}\right\rangle\right\|_{2} \\
& =\left[\sum_{j, k}\left\langle f, \hat{\psi}_{j, k}\right\rangle^{2}\right]^{\frac{1}{2}}=\left[\sum_{j, k} \frac{2^{2 j s}}{2^{2 j s}}\left\langle f, \hat{\psi}_{j, k}\right\rangle^{2}\right]^{\frac{1}{2}} \\
& \leq\left[\sum_{i, k} \frac{2^{2 j s}}{2^{2 p s}}\left\langle f, \hat{\psi}_{j, k}\right\rangle^{2}\right]^{\frac{1}{2}} \\
& =2^{-p s}\left[\sum_{j, k} 2^{2 j s}\left\langle f, \hat{\psi}_{j, k}\right\rangle^{2}\right]^{\frac{1}{2}} \leq 2^{-p s}\|f\|_{H \cdot[0,1]} .
\end{aligned}
$$

The same technique may be used to find error bolinds in the $H^{l}$ norm for $l \leq p$ :

$$
\begin{aligned}
\left\|f-P_{p} f\right\|_{H^{\prime}[0,1]} & =\left(\sum_{\substack{j \geq p \\
0 \leq k<2^{j}}}\left(1+2^{2 j l}\right)\left\langle f, \hat{\psi}_{j, k}\right\rangle^{2}\right)^{\frac{1}{2}} \\
& =\left(\sum_{j, k}\left(1+2^{2 j l}\right) \frac{\left(1+2^{2 j(s-l)}\right)}{\left(1+2^{2 j(s-l)}\right)}\left\langle f, \hat{\psi}_{j, k}\right\rangle^{2}\right)^{\frac{1}{2}} \\
& \leq\left(1+2^{2 p(s-l)}\right)^{-\frac{1}{2}}\left(\sum_{j, k}\left(1+2^{2 s j}\left\langle f, \hat{\psi}_{j, k}\right\rangle^{2}\right)^{\frac{1}{2}}\right. \\
& \leq 2^{-p(s-l)}\|f\|_{H^{\cdot}[0,1]} .
\end{aligned}
$$

These bounds are similar to those on Fourier series [11]. As illustrated in Figures 5 through 8, numerical results confirm the similarity of convergence rates for wavelets and Fourier methods. In these figures the dashed curve represents the wavelet case. Figures 5 and 6 show the $L^{2}$ difference of $P(x)$ and $S(x)$ at dyadic points with their wavelet and Fourier approximations, as a function of the number of interpolants. The curves show that the convergence rates are of the same order. Figure 5 also shows that for a lesser number of 
terms the two methods are comparable in capturing the features of a smooth pulse in the $L_{2}$, but as the number of terms is increased the Fourier method supercedes the wavelet method. Figure 6 shows that the Fourier method is marginally better than the wavelet method for the step function. The change in the slope of Figure 5 is due to the spectral shape of $P(x)$ : it is exponentially decaying while the step function has a spectrum that decays monotonically.

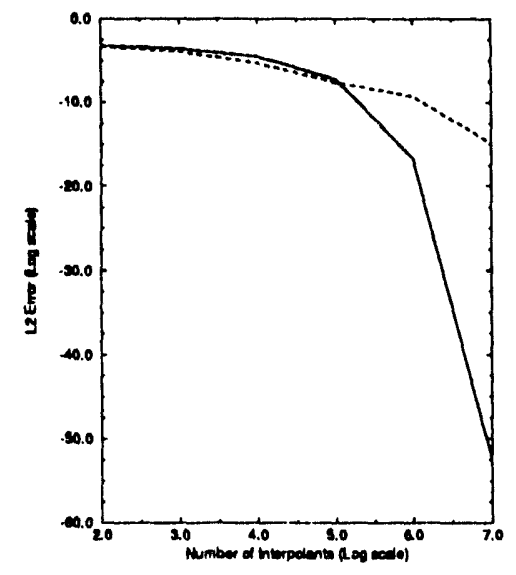

Figure 5. $L^{2}$ approximation error for $P(x)$. The dashed curve corresponds to the wavelet case.

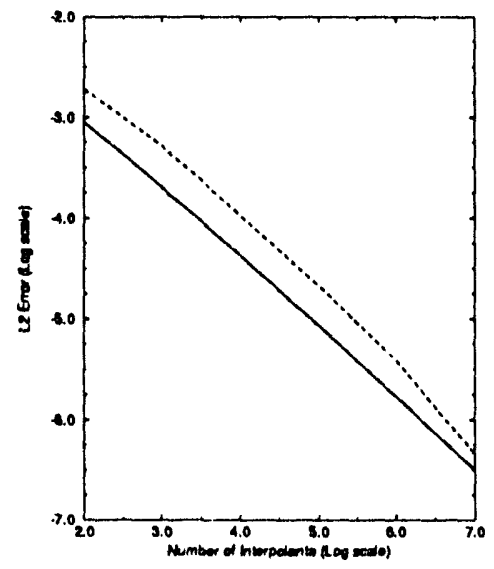

Figure 6. $L^{2}$ approximation error for $S(x)$. Dashed curve is the wavelet case.

Figures 7 and 8 show the $L_{1}$ approximation error. Figure 7 compares the error for the smooth pulse function $P(x)$ which shows marginally better characteristics for the wavelet case for a small number of interpolants, but for a larger number of modes, the Fourier method is clearly superior. As could have been expected, for the less smooth step function $S(x)$ the situation is the reverse. For a larger number of wavelet interpolants the $L_{1}$ error is smaller than the Fourier counterpart, as is evident in Figure 8. The results of this illustration squares well with the theoretical estimates provided elsewhere in this study. 


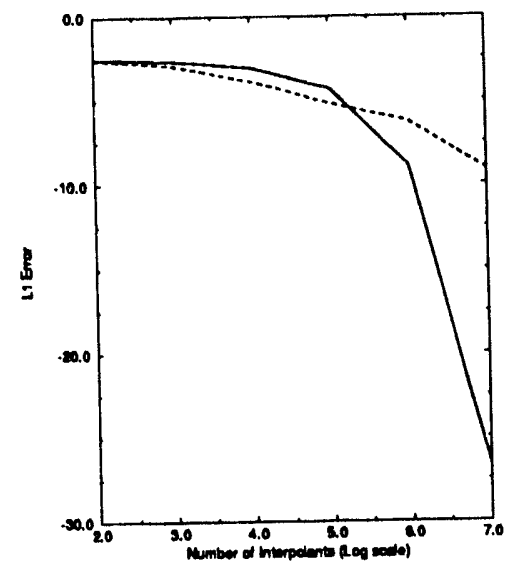

Figure 7. $L^{1}$ approximation error for $P(x)$. Dashed curve corresponds to the wavelet case.

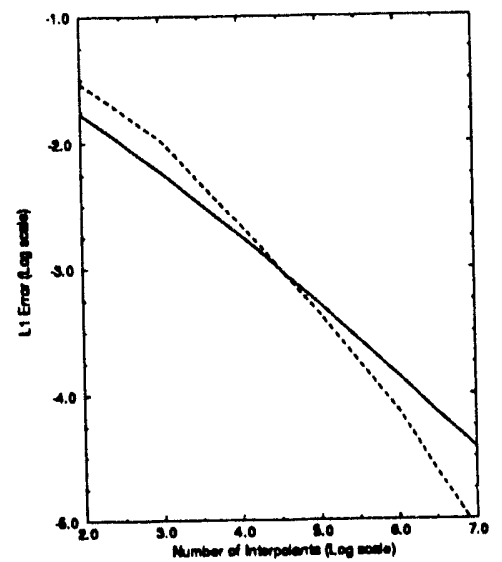

Figure 8. $L^{1}$ approximation error for $S(x)$. Dashed curve corresponds to the wavelet case.

\section{[4]. Methods for Computing Connection Coefficients.}

In wavelet applications, one often must represent operators in terms of wavelets [12]. An example of such an application is the Galerkin solution of differential equations. The formulation of solutions will require integrations of the form

$$
\int \varphi_{j, k_{0}}^{\left(d_{0}\right)} \varphi_{j, k_{1}}^{\left(d_{1}\right)} \cdots \varphi_{j, k_{n}}^{\left(d_{n}\right)}
$$

where $\varphi^{(d)}=\frac{d^{d} \varphi}{d x^{d}}$. This expression is an $n$-term connection coefficient and is denoted by $\Lambda_{k_{1}, k_{2}, \ldots, k_{n}}^{d_{1}, d_{2}, \ldots, d_{n}}$. Since $\varphi$ cannot be represented in close form for $N>2$ and, by construction, has limited regularity, analytic calculation of the integral is impossible, and numerical quadrature is often inaccurate. An alternative approach developed by Latto, Resnikoff, and Tenenbaum [7] circumvents some of the difficulty by exploiting the scaling relation and the moment condition to reduce the calculation to an eigenvector problem. Their method is designed for nonperiodic compactly supported wavelets. However, by invoking an extension of the earlier result regarding the equivalence of periodized and nonperiodized wavelets, one may infer that for $j \geq \log _{2}((N-1) n)$, the periodized case yields the same 
result as the nonperiodized case. Here we follow the procedure outlined in [7]. The 2-tuple case will be shown in detail; it is a simple exercise to extend the method to the $n$-tuple situation. Several tabulated connection coefficients are included in the appendix.

First, integration by parts is performed repeatedly on the above integral to obtain

$$
\Lambda_{k_{1}, k_{2}}^{d_{1}, d_{2}}=(-1)^{d_{1}} \Lambda_{k_{1}, k_{2}}^{0, d_{2}+d_{1}}
$$

where the compact support of the wavelets has been invoked. By changing variables, we further reduce the equations to

$$
\Lambda_{k_{1}, k_{2}}^{0, d} \Lambda_{0, k_{2}-k_{1}}^{0, d} \equiv \Lambda_{k_{2}-k_{1}}^{d}, \text { where } d=d_{1}+d_{2},
$$

From these relations it is clear that any 2 -tuple can be represented by a $\Lambda_{k}^{d}$.

To construct the eigenvector problem, fix $d$, then solve for $\left\{\Lambda_{k}^{d}\right\}_{0 \leq k<2^{j}}$ by creating a system of $2^{j}$ homogeneous relations in $\Lambda_{k}^{d}$ and enough inhomogeneous equations to reduce the dimension of the associated eigenspace to 1 . Although we are using the connectioncoefficient method for the nonperiodized case, we are computing them for the periodic case (by equivalence), which is where the bounds on $k$ come into play.

\subsection{Formation of Homogeneous Relations.}

Fix $d, j \in \mathbb{N}$, such that $\varphi_{j}^{(d)}$ is well defined. To simplify notation, denote $\varphi_{j, k}^{(d)} \equiv \Phi_{k}^{d}$. In [7] it is suggested, without proof, that this method also holds for the first $d$ for which $\Phi^{d}$ is discontinuous. For low-order differential equations, however, $D_{6}$ or $D_{8}$ wavelets should provide sufficient regularity. Since for every $0 \leq k<2^{j}$,

$$
\begin{aligned}
\Lambda_{k}^{d}=\int \Phi_{0}(x) \Phi_{k}^{d}(x) d x & =\int\left(\sum_{m=0}^{N-1} h_{m} \Phi_{m}(2 x)\right)\left(\sum_{l=0}^{N-1} h_{l} \Phi_{l+2 k}^{d}(2 x)\right) 2^{d} d(2 x) \\
& =2^{d} \sum_{m} \sum_{l} h_{m} h_{l} \int \Phi_{m}(2 x) \Phi_{l+2 k}^{d}(2 x) d(2 x) \\
& =2^{d} \sum_{m} \sum_{l} h_{m} h_{l} \int \Phi_{0}(\zeta) \Phi_{l+2 k-m}^{d}(\zeta) d \zeta ; \\
\text { thus, } \Lambda_{k}^{d} & =2^{d} \sum_{m=0}^{N-1} \sum_{l=0}^{N-1} h_{m} h_{l} \Lambda_{l+2 k-m}^{d}
\end{aligned}
$$

In the above discussion, the integration is over the real line and has been omitted to simplify notation.

This linear homogeneous system can be represented as

$$
\stackrel{A \lambda^{d}}{\rightarrow}=2^{-d} \stackrel{\lambda^{d}}{\rightarrow}
$$

where $\underline{\lambda}_{\rightarrow}^{d}=\left\{\Lambda_{k}^{d}\right\}_{0 \leq k<2^{j}}$. It is worth noting here that if one needs to compute an $n$-tuple connection coefficient for $j<\log _{2}((N-1) n)$, then the periodic scaling relation can be used to resolve each $\varphi_{j, k}$ into the sum of $\varphi_{j^{\prime}, k}$ 's with $j^{\prime} \geq \log _{2}((N-1) n)$. Thus, the reduction to the nonperiodic case is a universally applicable method. 


\subsection{Generating Inhomogeneous Relations.}

To generate the inhomogeneous equations, we must first assume $d \leq M-1$. The moment condition then guarantees that

$$
x^{d}=\sum_{l \in \mathbb{Z}} \tilde{M}_{l}^{d}
$$

where $\tilde{M}_{l}^{d}=\left\langle x^{d}, \varphi_{0, l}\right\rangle$. Setting $x=2^{j} \zeta$ and defining $M_{l}^{d}=\left\langle x^{d}, \Phi_{l}\right\rangle$, we have

$$
\tilde{M}_{l}^{d}=2^{d j} \dot{2}^{\frac{j}{2}}\left\langle\zeta^{d}, \Phi_{l}\right\rangle=2^{d j} \dot{2}^{\frac{j}{2}} M_{l}^{k} .
$$

This gives the relation

$$
\zeta^{d}=\sum_{l \in \mathbb{Z}} M_{l}^{d} \Phi_{l}(\zeta)
$$

which, when differentiated $d$ times, yields

$$
d !=\sum_{l \in \mathbb{Z}} M_{l}^{d} \Phi_{l}^{d}(\zeta)
$$

Multiplying by $\Phi_{0}^{0}$ and integrating, we obtain

$$
\sum_{l \in \mathbb{Z}} M_{l}^{d} \int \Phi_{0}^{0}(\zeta) \Phi_{l}^{d}(\zeta) d \zeta=d ! \int \varphi_{j, 0}(\zeta) d \zeta=d ! 2^{\frac{-i}{2}}
$$

Thus $\sum_{l} M_{l}^{d} \Lambda_{l}^{d}=d ! 2^{\frac{-j}{2}}$. The sum over $l$ is actually over $|l| \leq N-2$ since the $\varphi$ 's are compactly supported. Thus, by changing the indices of summation by $m=l+1+(N-2)$, the inhomogeneous equations are

$$
\begin{aligned}
& \sum_{m=1}^{2 N-3} \Lambda_{m}^{d} M_{m-1-(N-2)}^{d}, \\
& \text { with } M_{l}^{d}=2^{\frac{-j(2 d+1)}{2}} \tilde{M}_{l}^{d} .
\end{aligned}
$$

The linear system formed by the $2^{j}$ homogeneous equations and the above inhomogeneous equations has eigenspace dimension equal to 1 . Thus, all that remains to specify the system is to calculate $\tilde{M}_{l}^{d}$ :

$$
\begin{aligned}
\tilde{M}_{l}^{k}=\int x^{d} \varphi(x-l) d x & =\int(y+n)^{k} \varphi(y) d y \\
& =\int \sum_{j=0}^{k}\left(\begin{array}{c}
k \\
j
\end{array}\right) y^{j} n^{k-j} \varphi(y) d y \\
& =\sum_{j=0}^{k}\left(\begin{array}{l}
k \\
j
\end{array}\right) n^{k-j} \tilde{M}_{0}^{j} .
\end{aligned}
$$


Since $\tilde{M}_{l}^{0}=1$ is a previously stated property of the $\varphi$, the above relation is used to evaluate recursively $\tilde{M}_{l}^{d}$ for all $l$.

The linear system is now explicicly stated and guarantees exact values of $\Lambda_{k}^{d}$. Note that while the scaling relation, which is used to generate the homogeneous relations, exists for periodized wavelets, currently nothing is analogous to the moment condition which may be used to generate the necessary inhomogeneous equations. One possible approach is to use the scaling equation linked with the fact that $\hat{V}_{j}=$ \{constant functions $\}$ for $j \leq 0$ to find additional inhomogeneous relations. However, problems arise with relating $\int \hat{\varphi}_{j+1,0} d x$ to $\int \hat{\varphi}_{j, 0} d x$, since dilation does not commute with periodization. While this method could probably be worked out, the periodic case can always be reduced to an equivalent nonperiodic case for which the method is already well defined. Thus, to compute an $n$-term connection coefficient for periodized wavelets, one need only resolve the terms into $\hat{V}_{j}$, for some $j \geq \log _{2}(N-1) n$ and apply the above method. This approach takes full advantage of the equivalence of periodic and nonperiodic scaling functions and circumvents the need for a connection coefficient method particular to periodized wavelets. 


\section{APPENDIX}

Tables of Connection Coefficients.

Connection coefficients: 2-tuples for $p=0, N=6$.

$$
\begin{aligned}
& \Lambda_{1}^{0,1}=-3.4246575342471 D-04 \\
& \Lambda_{2}^{0,1}=-1.4611872146119 D-02 \\
& \Lambda_{3}^{0,1}=+0.14520547945205 \\
& \Lambda_{4}^{0,1}=-0.74520547945205 \\
& \Lambda_{5}^{0,1}=-3.2049276679778 D-15 \\
& \Lambda_{6}^{0,1}=+0.74520547945206 \\
& \Lambda_{7}^{0,1}=-0.14520547945205 \\
& \Lambda_{8}^{0,1}=+1.4611872146119 D-02 \\
& \Lambda_{9}^{0,1}=+3.4246575342476 D-04
\end{aligned}
$$

residual $=1.9680979936043 \mathrm{D}-16$

for the least-squares solution of the overdetermined system.

$\Lambda_{1}^{1,1}=+5.3571428571412 D-03$

$\Lambda_{2}^{1,1}=+0.11428571428572$

$\Lambda_{3}^{1,1}=-0.87619047619048$

$\Lambda_{4}^{1,1}=+3.3904761904762$

$\Lambda_{5}^{1,1}=-5.2678571428572$

$\Lambda_{6}^{1,1}=+3.3904761904762$

$\Lambda_{7}^{1,1}=-0.87619047619048$

$\Lambda_{8}^{1,1}=+0.11428571428571$

$\Lambda_{9}^{1,1}=+5.3571428571430 D-03$

residual $=1.1362438767648 \mathrm{D}-16$ 


$$
\begin{aligned}
& \Lambda_{1}^{2,0}=+5.3571428571412 D-03 \\
& \Lambda_{2}^{2,0}=+0.11428571428572 \\
& \Lambda_{3}^{2,0}=-0.87619047619048 \\
& \Lambda_{4}^{2,0}=+3.3904761904762 \\
& \Lambda_{5}^{2,0}=-5.2678571428572 \\
& \Lambda_{6}^{2,0}=+3.3904761904762 \\
& \Lambda_{7}^{2,0}=-0.87619047619048 \\
& \Lambda_{8}^{2,0}=+0.11428571428571 \\
& \Lambda_{9}^{2,0}=+5.3571428571430 D-03
\end{aligned}
$$

residual $=1.1362438767648 \mathrm{D}-16$

Tables of two-tuples and three-tuples shall be available as ascii files. Requests for connection coefficients tables should be made after August 1994. Send mail to wavelets@mcs.anl.gov. 


\section{REFERENCES}

1. C. Chui, An Introduction to Wavelets, Academic Press, New York, 1992.

2. I. Daubechies, Ten Lectures on Wavelets, Regional Conference Series in Applied Mathematics, vol. 61, SIAM, Philadelphia, 1992.

3. Y. Meyer, Ondelettes et Operateurs I, Editeurs des Sciences et des Arts, Hermann, 1990.

4. J. S. Xu and W. C. Shann, Galerkin-Wavelet Methods for Two-Point Boundary Value Problems, preprint, 1992.

5. G. Beylkin, R. Coifman and V. Rokhlin, Fast Wavelet Transforms and Numerical Algorithms I, Communications on Pure and Applied Mathematics 44 (1991), 141-183.

6. G. Battle, A Block Spin Construction of Ondelettes, Part I: Lemarié Functions, Communications in Mathematical Physics 110 (1987), 601-615.

7. A. Latto, H. L. Resnikoff and E. Tenenbaum, The Evaluation of Connection Coefficients of Compactly Supported Wavelets, preprint, 1991.

8. W. Press, S. Teukolsky, W. Vetterling and B. Flannery, Numerical Recipes in Fortran, in The Art of Scientific Computing, Cambridge University Press, Cambridge, 1992.

9. W. Sweldens and R. Piessens, Quadrature Formulae for the Calculation of the Wavelet Decomposition, preprint, 1991.

10. W. Rudin, Real and Complex Analysis, McGraw-Hill, Inc., New York, 1987.

11. C. Canuto, M. Y. Hussaini, A. Quarteroni and T. A. Zang, Spectral Methods in Fluid Dynamics, Springer Series in Computational Physics, Springer-Verlag, New York, 1988.

12. G. Beylkin, On the Representation of Operators in Bases of Compactly Supported Wavelets, SIAM Journal of Numerical Analysis 6 (1992), 1716-1740. 
Distribution for ANL-83/34

Internal :

J. M. Boumer (100)

F. Y. Fradin

G. K. Leaf (5)

G. W. Pieper

J. M. Restrepo (5)

R. L. Stevens

C. L. Wilkinson

TIS File

External:

DOE-OSTI, for distribution per UC-405 (54)

ANL-E Library (2)

All-H Library

Manager, Chicago Operations Office, DOE

Mathematics and Computer Science Division Review Committee:

H. H. Bledsoe, The University of Texas, Ausin

B. L. Buzbee, National Center for Atmospheric Research

J. G. Glimm, State University of New York at Stony Brook

M. T. Heath, University of Illinois, Urbana

E. F. Infante, University of Minnesota

D. O'Leary, University of Maryland

R. E. O'Malley, Rensselaer Polytechnic Institute

M. H. Schultz, Yale University

J. Cavallini, Department of Energy - Office of Scientific Computing

F. Howes, Department of Energy - Office of Scientific Computing

G. Schlossnagle, Johns Hopkins University (15) 

